\title{
Terrestrial Laser Scanning 3D as a useful method for monitoring of urban areas endangered by landslides
}

\author{
Mirosława Bazarnik \\ Cracow University of Technology, Faculty of Civil Engineering; ul. Warszawska 24, 31-155 Krakow, Poland; \\ e-mail:mbazarnik@pk.edu.pl \\ (C) 2015 Authors. This is an open access publication, which can be used, distributed and reproduced in any medium according \\ to the Creative Commons CC-BY 4.0 License requiring that the original work has been properly cited.
}

Landslides are a serious problem in Poland in recent years. Starting from catastrophic landslide events in 1990s systematically is increased risk of landslides hazard on urbanized areas. However, many of Polish landslides are slow-moving, so potential terrain instability is often difficult to observe or it is easy to neglect (Mrozek et al. 2013). In the analysis of landslides (predicting, monitoring and alerting for early warning, monitoring for change detection, etc.) a variety of geomatic techniques can be used. One of the most innovative is Terrestrial Laser scanner 3D. Terrestrial Laser Scanning, usually adopted in industry, could be successfully applied for deformation monitoring of the landslide. This technique give a great contribution to the knowledge of both the surface shape and the kinematics of landslides by providing data which are used by geologists, geomorphologists and geotechnics for interpreting the phenomenon. Such a methodology offers the advantages typical of noncontact techniques, and moreover permits to collect in short time dense 3D point clouds over the surface of interest, to record a perspective image (intensity data and sometimes RGB data), it doesn't requires necessarily deployment of reflectors, and it permits immediately and easily to take measurements between points (Bitelli et al. 2004).

The acquired points clouds data have to be connected together in order to reconstruct the continuous surface of the landslide, so they are auto- matically aligned during the survey and merged by the software, which permits to create polygonal surfaces and to map the surface with textures using digital photos obtained during the scanning by a digital semi-metric camera placed over the laser instrument (Barbarella \& Fiani 2012).

The Terrestrial Laser Scanner allows us to survey in a very fast, efficient and precise way. It could be very useful because of their ability to describe in details the geometry of objects. In the analysis of the temporal evolution of landslides and of related hydrogeological hazards, terrestrial laser scanning (TLS) seems to be a very suitable technique for morphological description and displacement analysis. Monitoring in general can be regarded as the regular observation and recording of activities taking place in a certain structure. It is a process of gathering information on all aspects of the object (Pilecka \& Bazarnik 2015). Deformation monitoring and gathering measured values is a major component for further calculation of soil and rock stability, deformation analysis, prediction and alert.

\section{REFERENCES}

Barbarella M. \& Fiani M., 2012. Landslide monitoring using terrestrial laser scanner: georeferencing and canopy filtering issues in a case study. International Archives of the Photogrammetry, Remote Sensing and Spatial Information Sciences, 39 (5B), 157-162. 
Bitelli G., Dubbini M. \& Zanutta A., 2004. Terrestrial laser scanning and digital photogrammetry techniques to monitor landslide bodies. International Archives of the Photogrammethry, Remote Sensing and Spatial Information Science, 38 (7B), 246-251.

Mrozek T., Wójcik A., Zimnal Z. \& Grabowski D., 2013. Landslide Inventory at 1:10,000 Scale in Poland: Benefits and Dilemmas of a National Project. [in:] Margottini C., Canuti P. \& Sassa K. (Eds), Landslide Science and Practice, Springer, 51-55.

Pilecka E. \& Bazarnik M., 2015. Application of terrestrial laser scanner for monitoring the railway infrastructure threatened by landslides [in press]. 